\title{
Integro-Differential Polynomials and Operators
}

\author{
Markus Rosenkranz and Georg Regensburger \\ Johann Radon Institute for Computational and Applied Mathematics (RICAM) \\ Austrian Academy of Sciences \\ Altenbergerstraße 69 \\ A-4040 Linz, Austria \\ \{markus.rosenkranz,georg.regensburger\}@oeaw.ac.at
}

\begin{abstract}
We propose two algebraic structures for treating integral operators in conjunction with derivations: The algebra of integro-differential polynomials describes nonlinear integral and differential operators together with initial values. The algebra of integro-differential operators can be used to solve boundary problems for linear ordinary differential equations. In both cases, we describe canonical/normal forms with algorithmic simplifiers.
\end{abstract}

\section{Categories and Subject Descriptors}

I.1.1 [Symbolic and Algebraic Manipulation]: Expressions and Their Representation-simplification of expressions; I.1.2 [Symbolic and Algebraic Manipulation]: Algorithms - algebraic algorithms

\section{General Terms}

Theory, Algorithms

\section{Keywords}

Integral operators, integro-differential algebras, noncommutative Gröbner bases, Green's operators, linear boundary value problems

\section{INTRODUCTION}

While differential operators are studied extensively in symbolic computation, this cannot be asserted about integral operators. In the former case, one uses two fundamental structures for transferring analysis to algebra: "differential operators" and "differential polynomials"; both of these can act on suitable function spaces (the former linearly and the latter nonlinearly). In this paper, we propose two analogous algebraic structures for treating integral operators (along with differential operators): "integro-differential operators"

*This work was supported by the Austrian Science Fund (FWF) under the SFB grant F1322. and "integro-differential polynomials"; both of these are extensions of the corresponding differential structures. In Section 2 , we review some notions about integro-differential algebras needed for these constructions.

The integro-differential polynomials are introduced here for the first time. Their construction is explained in Section 3, the computational approach in Section 4. While modeling nonlinear integral operators, the most important use of the integro-differential polynomial ring $\mathcal{F}\{u\}$ over a given integro-differential algebra $\mathcal{F}$ is probably to describe extensions of integro-differential algebras in a constructive fashion. In practice, one can start with the integro-differential algebra $\mathcal{F}_{0}$ of exponential polynomials, adjoin a solution of differential equations (with initial values) by passing to a quotient $\mathcal{F}_{1}$ of $\mathcal{F}_{0}\{u\}$, and iterate this procedure.

The notion of integro-differential operators has been introduced in [23], where it is used for multiplying and factoring BVPs (= linear boundary value problems for ordinary differential equations). In fact, one of the main applications of integro-differential operators is that they describe the differential equation, boundary conditions and solution operator (Green's operator) of a BVP in uniform language. In [23] we have constructed a monoid on BVPs isomorphic to the compositional structure of their Green's operators, studied in [21] from an abstract viewpoint. In this paper, we will review their construction and main properties in Section 5 and focus on computational aspects in Section 6 .

For both integro-differential polynomials and operators, the crucial instrument for an algorithmic treatment is of course the usage of standard representatives, but they arise in fairly different contexts: In the former case, where we prefer to speak of canonical forms, we employ tools from universal algebra to build a canonical simplifier for the appropriate polynomial concept. In the latter case, where we shall use word normal forms, our approach is to construct a confluent rewrite system (equivalently: a noncommutative Gröbner basis).

\section{INTEGRO-DIFFERENTIAL ALGEBRAS}

Our starting point is a commutative differential algebra $(\mathcal{F}, \partial)$ over a field $K$, so $\partial: \mathcal{F} \rightarrow \mathcal{F}$ is a $K$-linear map satisfying the Leibniz rule

$$
\partial(f g)=f \partial(g)+g \partial(f) .
$$


For convenience, we may assume $K \leq \mathcal{F}$, and we write $f^{\prime}$ as a shorthand for $\partial(f)$. Furthermore, we will assume that $K$ has characteristic zero and $\mathbb{Q} \leq K$, hence $\mathcal{F}$ is what is sometimes called a Ritt algebra [14, p. 12]. The algebra of differential operators over $\mathcal{F}$ is denoted by $\mathcal{F}[\partial]$ as in [27].

For inhomogeneous differential equations $T u=f$ with $T \in$ $\mathcal{F}[\partial]$, the solution operators (mapping $f \in \mathcal{F}$ to $u \in \mathcal{F}$ ) are integral operators. The simplest equation is $u^{\prime}=f$, and its solution operators $\int$ are exactly the sections (i.e. $K$-linear right inverses) of the differential operator $\partial$ so that

$$
\partial \int=1 \text {. }
$$

Note that derivations need not have sections (for example in the algebra of univariate differential polynomials, the indeterminate cannot be a derivative).

The characterization of sections follows from Linear Algebra, see [19, p. 17] or [21]: Every section $\int: \mathcal{F} \rightarrow \mathcal{F}$ of the derivation $\partial: \mathcal{F} \rightarrow \mathcal{F}$ corresponds to a unique projector $P: \mathcal{F} \rightarrow \mathcal{F}$ with

$$
P=1-\int \partial
$$

and to a unique direct sum decomposition $\mathcal{F}=\mathcal{C} \dot{+} \mathcal{I}$ of $K$-vector spaces with

$$
\mathcal{C}=\operatorname{Ker}(\partial)=\operatorname{Im}(P) \quad \text { and } \quad \mathcal{I}=\operatorname{Im}\left(\int\right)=\operatorname{Ker}(P) .
$$

Moreover, if $\int$ is any fixed section of $\partial$, every projector $P$ with $\operatorname{Im}(P)=\operatorname{Ker}(\partial)$ induces a section $(1-P) \int$, and every section of $\partial$ arises uniquely in this way.

We refer to the elements of $\mathcal{I}=\operatorname{Im}\left(\int\right)$ as initialized (with respect to $\int$ ), while those of $\mathcal{C}=\operatorname{Ker}(\partial)$ are usually called the constants (with respect to $\partial$ ).

As a standard example, we take $\mathcal{F}=C^{\infty}[a, b]$ where differentiability in the endpoints is understood in the sense onesided derivatives. The initialized functions are those that can be written as $F(x)=\int_{\alpha}^{x} f(\xi) d \xi$ for $f \in C^{\infty}[a, b]$ and an initialization point $\alpha \in[a, b]$; hence $F$ is the unique antiderivative of $f$ that fulfills the initial condition $F(\alpha)=0$.

For solving inhomogeneous differential equations of higher order, one must expect to iterate the section $\int$. While this would in general lead to nested integrals, we know from the classical $C^{\infty}$ setting that the Green's operator can always be expressed via the Green's function [26] by a single integration. To capture this behavior, we need an identity for resolving nested integrals (eventually leading to the rewrite rule for $\int f \int$ in Table 1). Such an identity is given by the so-called Baxter axiom (of weight zero), asserting

$$
\int f \cdot \int g=\int f \int g+\int g \int f
$$

for all $f, g \in \mathcal{F}$. Note that we apply the following convention in this paper: An integral like $\int f \int g$ should be interpreted as $\int\left(f \int g\right)$, unless we use $\cdot$ as on the left-hand side above.

Obviously (4) is an algebraic version of integration by parts, written in a way that does not involve the derivation. (For the integro-differential polynomials, the role of the Baxter axiom is more subtle: From the left to right, it "flattens" products of nested integrals; in the other direction, it is used for "integrating out" coefficient functions - see Section 4.) A weight-zero Baxter algebra $\left(\mathcal{F}, \int\right)$ is then a $K$-algebra $\mathcal{F}$ with a $K$-linear operation $\int$ fulfilling the Baxter axiom (4); we refer to $[11,2,24]$ for more details.

What we shall actually use is the differential Baxter axiom, which requires

$$
\int f g=f \int g-\int f^{\prime} \int g
$$

for all $f, g \in \mathcal{F}$. Note that this is what most people do when they actually apply integration by parts. Variant (4) follows immediately by substituting $\int f$ for $f$ in (5), and often both versions are equivalent (see after Definition 11).

We can also characterize what makes the differential Baxter axiom stronger than the pure one: A section $\int$ of $\partial$ fulfills the differential Baxter axiom (5) iff it fulfills the pure Baxter axiom (4) and the homogeneity condition

$$
\int c f=c \int f
$$

for all $c \in \mathcal{C}$ and $f \in \mathcal{F}$. In fact, (6) implies that $\int: \mathcal{F} \rightarrow \mathcal{F}$ is $\mathcal{C}$-linear and not only $K$-linear.

We refer to [23] for the proof of the equivalence and for an example of a differential algebra with a section that satisfies the pure Baxter axiom but not its differential form. To exclude such cases we will insist that integral operators must satisfy the differential Baxter axiom.

DeFinition 1. Let $\mathcal{F}$ be a differential algebra over a field $K$. A section $\int$ of $\partial$ is called an integral if it satisfies the differential Baxter axiom (5). In this case, we call $\left(\mathcal{F}, \partial, \int\right)$ an integro-differential algebra.

As an example, take $\mathcal{F}=C^{\infty}[a, b]$ with its usual derivation $\partial$ and integral operators

$$
\int^{*}: f \mapsto \int_{a}^{x} f(\xi) d \xi \quad \text { and } \quad \int_{*}: f \mapsto \int_{x}^{b} f(\xi) d \xi .
$$

Then both $\left(\mathcal{F}, \partial, \int^{*}\right)$ and $\left(\mathcal{F}, \partial,-\int_{*}\right)$ are integro-differential algebras. By contrast, the operator

$$
f \mapsto \int_{a}^{b} \int_{\tau}^{x} f(\xi) d \xi d \tau
$$

is just a section for $\partial$, but not an integral.

In the above example, the projectors $P^{\star}: f \mapsto f(a)$ and $P_{\star}: f \mapsto f(b)$ corresponding to the respective integral operators $\int^{*}$ and $\int_{*}$ are multiplicative (see (7) below), whereas the projector $\int_{a}^{b}$ for the third operator is not. This is true in general - we can characterize integrals by their projectors or images as detailed in [23]: A section $\int: \mathcal{F} \rightarrow \mathcal{F}$ of the derivation $\partial: \mathcal{F} \rightarrow \mathcal{F}$ is an integral iff $\mathcal{I}=\operatorname{Im}\left(\int\right)$ is an ideal of $\mathcal{F}$ iff $P=1-\int \partial$ is multiplicative, meaning

$$
P(f g)=P(f) P(g)
$$

for all $f, g \in \mathcal{F}$. Using the homogeneity condition (6), this implies also

$$
\int f g^{\prime}=f g-\int f^{\prime} g-P(f) P(g)
$$

as an equivalent formulation (corresponding to the rewrite rule for $\int f \partial$ in Table 1 ) of the differential Baxter axiom (5). 
Similar structures are introduced under the name differential Rota-Baxter algebras in the recent article [12]. A crucial difference is that they only require the section axiom (2) for connecting derivation and integral, but not the differential Baxter axiom (5). They construct free objects in more general categories where the algebras are over unital commutative rings rather than fields, they may be noncommuative, and the weight can be an arbitrary scalar.

\section{THE ALGEBRA OF INTEGRO- DIFFERENTIAL POLYNOMIALS}

In this section, we introduce the algebra of integro-differential polynomials obtained by adjoining one indeterminate function to an integro-differential algebra. This is a special case of the general construction of polynomials in universal algebra. See for example [1] for the basic notions in universal algebra that we use in the following and $[7,13,16]$ for details on polynomials in universal algebra.

The idea of the construction is as follows. Let $\mathcal{V}$ be a variety defined by a set $E$ of identities or "laws" over a signature $\Sigma$. Let $A$ be a fixed "coefficient domain" from the variety $\mathcal{V}$, and let $X$ be a set of "variables" or "indeterminates". Then all terms in the signature $\Sigma$ with constants (henceforth called "coefficients") in $A$ and variables in $X$ represent the same polynomial if their equality can be derived in finitely many steps from the identities in $E$ and the operations in $A$. The set of all such terms $\mathcal{T}_{\Sigma}(A \cup X)$ modulo this congruence $\equiv$ is an algebra in $\mathcal{V}$, called the polynomial algebra (for $\mathcal{V}$ ) in $X$ over $A$, denoted by $A_{\mathcal{V}}[X]$.

The polynomial algebra $A_{\mathcal{V}}[X]$ contains $A$ as a subalgebra, and $A \cup X$ is a generating set. As in the case of polynomials for commutative rings, we have the substitution homomorphism in general polynomial algebras. Let $B$ be an algebra in $\mathcal{V}$. Then given a homomorphism $\varphi_{1}: A \rightarrow B$ and a map $\varphi_{2}: X \rightarrow B$, there exists a unique homomorphism

$$
\varphi: A \mathcal{V}[X] \rightarrow B
$$

such that $\varphi(a)=\varphi_{1}(a)$ for all $a \in A$ and $\varphi(x)=\varphi_{2}(x)$ for all $x \in X$.

In order to compute with polynomials one can use an effective canonical simplifier [7], that is, a computable map

$$
\sigma: \mathcal{T}_{\Sigma}(A \cup X) \rightarrow \mathcal{T}_{\Sigma}(A \cup X)
$$

such that

$$
\sigma(T) \equiv T \quad \text { and } \quad S \equiv T \Rightarrow \sigma(S)=\sigma(T)
$$

for all terms $S, T \in \mathcal{T}_{\Sigma}(A \cup X)$. The representatives in $\mathcal{R}:=\operatorname{Im}(\sigma)$ are called canonical forms. Canonical simplifiers correspond uniquely to so-called systems of canonical forms, i.e. a set of terms

$$
\mathcal{R} \subseteq \mathcal{T}_{\Sigma}(A \cup X)
$$

such that for every $T \in \mathcal{T}_{\Sigma}(A \cup X)$ one can compute a canonical form $R \in \mathcal{R}$ with $T \equiv R$ and such that $R \neq \tilde{R} \Rightarrow R \neq \tilde{R}$ for $R, \tilde{R} \in \mathcal{R}$. In other words, for every polynomial in $A_{\mathcal{V}}[X]$ represented by a term $T$ one can compute a term $R \in \mathcal{R}$ representing the same polynomial, with different terms in $\mathcal{R}$ representing different polynomials, see [16, p. 23].
As a well-known example take the polynomial ring $R[x]$ in one indeterminate $x$ over a commutative ring $R$. The set of all terms of the form $a_{n} x^{n}+\ldots+a_{0}$ with coefficients $a_{i} \in R$ and $a_{n} \neq 0$ together with 0 is a system of canonical forms for $R[x]$. One usually defines the polynomial ring directly in terms of these canonical forms. Polynomials for groups, bounded lattices and Boolean algebras are discussed in [16] along with systems of canonical forms.

Let us now consider the variety $\mathcal{V}$ of integro-differential algebras. Its signature $\Sigma$ contains (besides the ring operations): the derivation $\partial$, the integral $\int$, the family of unary "scalar multiplications" $(\cdot \lambda)_{\lambda \in K}$; for convenience we also include the projection $P$. The identities $E$ are (besides those of a $K$-algebra and the $K$-linearity of the operators $\partial, \int, P$ ): the Leibniz rule (1), the section axiom (2), the definition of the projection (3), and the differential Baxter axiom (5).

DeFinition 2. Let $\mathcal{F}$ be an integro-differential algebra. Then $\mathcal{F}_{\mathcal{V}}[u]$ is called the algebra of integro-differential polynomials in $u$ over $\mathcal{F}$ and denoted it by $\mathcal{F}\{u\}$ in analogy to differential polynomials.

We will also use the following identities following from $E$ and describing the basic interactions between the operations in $\mathcal{F}$ : the pure Baxter axiom (4), the multiplicativity of the projection $(7)$, the identities

$$
P^{2}=P, \quad \partial P=0, \quad P \int=0, \quad \int P(f) g=P(f) \int g,
$$

and the variant (8) of the differential Baxter axiom connecting all three operations. Moreover, we use also the shuffle identity $[25,20]$ obtained from iterating the Baxter axiom

$$
\int f_{1} \int \ldots \int f_{m} \cdot \int g_{1} \int \ldots \int g_{n}=\sum \int h_{1} \int \ldots \int h_{m+n},
$$

where the sum ranges over all shuffles of $\left(f_{1}, \ldots, f_{m}\right)$ and $\left(g_{1}, \ldots, g_{n}\right)$. By construction of the polynomial algebra, all these identities hold also for $\mathcal{F}\{u\}$.

We will use $f, g$ for denoting coefficients in $\mathcal{F}$ and $V$ for terms in $\mathcal{T}_{\Sigma}(\mathcal{F} \cup\{u\})$. As for differential polynomials, we write $u_{n}$ for the $n$th derivative of $u$. We use the multi-index notation

$$
u^{\beta}=\prod_{i=0}^{\infty} u_{i}^{\beta_{i}}
$$

for a sequence $\beta$ in $\mathbb{N}$ with only finitely many nonzero entries. The order of a differential monomial $u^{\beta}$ is the highest derivative appearing in $u^{\beta}$ or $-\infty$ if $\beta=0$. Moreover, we write $V(0)$ for $P(V)$ and

$$
u(0)^{\alpha}=\prod_{i=0}^{\infty} u_{i}(0)^{\alpha_{i}} .
$$

for a multi-index $\alpha$.

\section{CANONICAL FORMS FOR INTEGRO- DIFFERENTIAL POLYNOMIALS}

Our goal is to find a system of canonical forms for integrodifferential polynomials. As a first step, we describe a system of terms that is sufficient for representing every polynomial, but not in a unique (canonical) way. 
LEMma 3. Every polynomial in $\mathcal{F}\{u\}$ can be represented by a finite sum of terms of the form

$$
f u(0)^{\alpha} u^{\beta} \int f_{1} u^{\gamma_{1}} \int \ldots \int f_{n} u^{\gamma_{n}},
$$

where each multi-index as well as $n$ may be zero.

Proof. By induction on the structure of terms, using the identities of integro-differential algebras and the above mentioned consequences (except the differential variants of the Baxter axiom).

Note that for terms only involving the derivation, (11) gives already the usual canonical form for differential polynomials. With the aid of Lemma 3, we can now determine the constants in $\mathcal{F}\{u\}$.

Proposition 4. Every constant in $\mathcal{F}\{u\}$ can be represented as a finite sum $\sum_{\alpha} c_{\alpha} u(0)^{\alpha}$ with constants $c_{\alpha}$ in $\mathcal{F}$.

Proof. By the identity $\int \partial=1-P$, a term $V$ represents a constant in $\mathcal{F}\{u\}$ iff $P(V) \equiv V$. Since $V$ is congruent to a finite sum of terms of the form $(11)$ and since $\operatorname{Im}(P)=\mathcal{C}$, the identities for $P$ imply that $V$ is congruent to a finite sum of terms of the form $c_{\alpha} u(0)^{\alpha}$.

It is immediately clear that terms of the form (11) cannot be canonical forms for general integro-differential polynomials since for example $f \int g u$ and $\lambda^{-1} f \int \lambda g u$ with $\lambda \in K$ represent the same polynomial. This can be solved by choosing a basis $\mathcal{B}$ for $\mathcal{F}$ containing 1 .

A second problem for canonical forms comes from the fact that we can integrate certain differential polynomials using integration by parts (8). For example, the terms $\int f u^{\prime}$ and $f u-\int f^{\prime} u-f(0) u(0)$ represent the same polynomial. More generally, we have the following identity.

\section{LEMma 5. We have}

$$
\begin{aligned}
& \int V u_{k}^{\beta_{k}} u_{k+1} \\
& \equiv \frac{1}{\beta_{k}+1}\left(V u_{k}^{\beta_{k}+1}-\int V^{\prime} u_{k}^{\beta_{k}+1}-V(0) u_{k}(0)^{\beta_{k}+1}\right)
\end{aligned}
$$

where $k, \beta_{k} \geq 0$.

Proof. Using (8) and the Leibniz rule, we see that

$$
\begin{aligned}
& \int V u_{k}^{\beta_{k}} u_{k+1}=\int\left(V u_{k}^{\beta_{k}}\right)\left(u_{k}\right)^{\prime} \\
\equiv & V u_{k}^{\beta_{k}+1}-\int V^{\prime} u_{k}^{\beta_{k}+1}-\beta_{k} \int V u_{k}^{\beta_{k}} u_{k+1}-V(0) u_{k}(0)^{\beta_{k}+1},
\end{aligned}
$$

and the equation follows.

In particular, if $V=f u_{0}^{\beta_{0}} \ldots u_{k-1}^{\beta_{k-1}}$, then $V^{\prime}$ and hence also the right-hand side of (12) contains only differential monomials with order at most $k$. So if the highest derivative in the differential monomial $u^{\beta}$ of order $k+1$ appears linearly, the term $\int f u^{\beta}$ is congruent to a sum of terms involving only differential monomials of order at most $k$. This motivates the following classification of differential monomials; confer also $[4,10]$.
Definition 6. A monomial (11), with $u^{\beta}$ having order $k$, is said to have depth $n$ and order $k$. It is called quasiconstant if $\beta=0$, quasilinear if $k>0$ and the highest derivative appears linearly; otherwise it is called functional.

Definition 7. We write $\mathcal{R}$ for the set of all $K$-linear combinations of terms of the form

$$
b u(0)^{\alpha} u^{\beta} \int b_{1} u^{\gamma_{1}} \int \ldots \int b_{n} u^{\gamma_{n}},
$$

where $b, b_{1}, \ldots, b_{n} \in \mathcal{B}$, the multi-indices $\alpha, \beta$ as well as $n$ may be zero, and $u^{\gamma_{1}}, \ldots, u^{\gamma_{n}}$ are functional.

As we will see, $\mathcal{R}$ forms a system of canonical forms for $\mathcal{F}\{u\}$. The easier part of this claim is that every polynomial has such a representation.

Proposition 8. Every polynomial in $\mathcal{F}\{u\}$ can be represented by a term in $\mathcal{R}$.

Proof. Using basis expansions and the $K$-linearity of the integral, we can represent with Lemma 3 every polynomial in $\mathcal{F}\{u\}$ as a $K$-linear combination of terms of the form

$$
b u(0)^{\alpha} u^{\beta} \int b_{1} u^{\gamma_{1}} \int \ldots \int b_{n} u^{\gamma_{n}},
$$

where the multi-indices and $n$ can also be zero.

With basis expansions and the identity

$$
\int f \int V \equiv \int f \cdot \int V-\int V \int f
$$

coming from the pure Baxter axiom (4), we can achieve that every multi-index $\gamma_{k}$ in (14) is nonzero (induction on depth). Using Lemma 5 , one sees that a term $\int b_{1} u^{\gamma_{1}}$ is congruent to a sum of terms involving only integral terms with functional differential monomials (induction on order). Finally one shows (induction on depth and order) that this also holds for terms of the form

$$
\int b_{1} u^{\gamma_{1}} \int \ldots \int b_{n} u^{\gamma_{n}} .
$$

The proposition then follows by basis expansions and the $K$-linearity of the integral.

It remains to show that each term in $\mathcal{R}$ represents a different polynomial. To this end, let $\langle\mathcal{R}\rangle$ be the free vector space over the set of terms (13). In order to distinguish the basis vectors of $\langle\mathcal{R}\rangle$ from the corresponding terms in $\mathcal{R}$, we denote them by

$$
\left\langle b u(0)^{\alpha} u^{\beta} \int b_{1} u^{\gamma_{1}} \int \ldots \int b_{n} u^{\gamma_{n}}\right\rangle .
$$

If $b, b_{1}, \ldots, b_{n}$ are no basis vectors, (15) is to be understood as an abbreviation for the corresponding basis expansion. We equip the free vector space $\langle\mathcal{R}\rangle$ with the structure of an integro-differential algebra. The operations are defined on the basis vectors mimicking the corresponding operations in $\mathcal{T}_{\Sigma}(\mathcal{F} \cup\{u\})$, and reducing to congruent terms in $\mathcal{R}$.

The multiplication in $\langle\mathcal{R}\rangle$ is introduced in stages. Let $J$ and $\tilde{J}$ range over pure integral terms $\int b_{1} u^{\gamma_{1}} \int \ldots \int b_{n} u^{\gamma_{n}}$, 
including 1 for $n=0$. The product of a term $\left\langle b u(0)^{\alpha} u^{\beta}\right\rangle$ with a general term $\left\langle\tilde{b} u(0)^{\tilde{\alpha}} u^{\tilde{\beta}} \tilde{J}\right\rangle$ is defined as

$$
\left\langle b \tilde{b} u(0)^{\alpha+\tilde{\alpha}} u^{\beta+\tilde{\beta}} \tilde{J}\right\rangle .
$$

Corresponding to the shuffle identity (10), we define the product $\left\langle\int b u^{\gamma} J\right\rangle\left\langle\int \tilde{b} u^{\tilde{\gamma}} \tilde{J}\right\rangle$ of pure integrals recursively as

$$
\left\langle\int b u^{\gamma}\right\rangle \star\langle J\rangle\left\langle\int \tilde{b} u^{\tilde{\gamma}} \tilde{J}\right\rangle+\left\langle\int \tilde{b} u^{\tilde{\gamma}}\right\rangle \star\langle\tilde{J}\rangle\left\langle\int b u^{\gamma} J\right\rangle,
$$

where $\star$ denotes the operation of nesting integrals (multiplication binds stronger than $\star$ ); the base case is given by the neutral element 1 . With this product, the pure integral terms form a subalgebra isomorphic to the shuffle algebra so that $\cdot$ is associative and commutative. Finally, the product of two general basis vectors $\left\langle b u(0)^{\alpha} u^{\beta} J\right\rangle$ and $\left\langle\tilde{b} u(0)^{\tilde{\alpha}} u^{\tilde{\beta}} \tilde{J}\right\rangle$ is given by multiplying $\left\langle b u(0)^{\alpha} u^{\beta}\right\rangle\left\langle\tilde{b} u(0)^{\tilde{\alpha}} u^{\tilde{\beta}}\right\rangle$ with $\langle J\rangle\langle\tilde{J}\rangle$.

The derivation of basis vector is defined through the Leibniz rule, using also the identities $\partial P=0, \partial \int=1$ and basis expansions.

The integral of a basis vector is defined recursively (first by depth and then by order), based on the classification of Definition 6. In the quasiconstant case, we define

$$
\int\left\langle b u(0)^{\alpha} J\right\rangle=\left\langle\int b\right\rangle\left\langle u(0)^{\alpha} J\right\rangle-\int\left\langle J^{\prime}\right\rangle \int\left\langle b u(0)^{\alpha}\right\rangle,
$$

where $J^{\prime}$ is $J$ with the integral removed (zero for $J=1$ ). For a quasilinear basis vector

$$
\left\langle b u(0)^{\alpha} V u_{k}^{\beta_{k}} u_{k+1} J\right\rangle \text { with } V=u_{0}^{\beta_{0}} \ldots u_{k-1}^{\beta_{k-1}},
$$

we set $s=\beta_{k}+1$ and define the integral by

$$
\begin{aligned}
& s \int\left\langle b u(0)^{\alpha} V u_{k}^{\beta_{k}} u_{k+1} J\right\rangle \\
& =\left\langle b u(0)^{\alpha} V u_{k}^{s} J\right\rangle-\left\langle u(0)^{\alpha}\right\rangle \int\langle b V J\rangle^{\prime}\left\langle u_{k}^{s}\right\rangle-\left\langle b V u^{\alpha} u_{k}^{s} J\right\rangle(0) ;
\end{aligned}
$$

the third summand is absent unless $J=1$.

In the functional case, we use

$$
\int\left\langle b u(0)^{\alpha} u^{\beta} J\right\rangle=\left\langle u(0)^{\alpha} \int b u^{\beta} J\right\rangle,
$$

as a definition for the integral.

For showing that $\langle\mathcal{R}\rangle$ is an integro-differential algebra, we have to verify the axioms: First of all we see that it is a commutative $K$-algebra by our previous remark about the shuffle product. The Leibniz rule and the section axiom follow immediately from the definition. The only difficult task is to prove the differential Baxter axiom. An easy calculation shows that

$$
\int\left\langle u(0)^{\alpha}\right\rangle\langle R\rangle=\left\langle u(0)^{\alpha}\right\rangle \int\langle R\rangle .
$$

Proposition 4 then implies that $\int$ is homogeneous over the constants in $\langle\mathcal{R}\rangle$. By the observation before (6), it suffices therefore to verify the pure Baxter axiom. The proof is lengthy (using inductions over depth and order, with case distinctions according to the definition of the integral) and will be presented in a subsequent publication.

Proposition 9. With the operations defined as above, $\langle\mathcal{R}\rangle$ is an integro-differential algebra.
The integro-differential algebra $\langle\mathcal{R}\rangle$ provides the key for showing that all terms in $\mathcal{R}$ represent different polynomials of $\mathcal{F}\{u\}$.

ThEOREM 10. The terms in $\mathcal{R}$ constitute a system of canonical forms for $\mathcal{F}\{u\}$, provided that basis expansion in $\mathcal{F}$ is computable.

Proof. Since $\langle\mathcal{R}\rangle$ is an integro-differential algebra, there exists a unique substitution homomorphism

$$
\varphi: \mathcal{F}\{u\} \rightarrow\langle\mathcal{R}\rangle
$$

such that $\varphi(f)=\langle f\rangle$ for all $f \in \mathcal{F}$ and $\varphi(u)=\langle u\rangle$. Let

$$
\pi: \mathcal{R} \rightarrow \mathcal{F}\{u\}
$$

denote the restriction of the canonical epimorphism associated with $\equiv$. Then $\varphi \circ \pi$ is injective since it maps $R \in \mathcal{R}$ to $\langle R\rangle \in\langle\mathcal{R}\rangle$ and surjective by Proposition 8 . We conclude that $\pi$ is also bijective, so $\mathcal{R}$ is indeed a system of canonical forms.

\section{THE ALGEBRA OF INTEGRO- DIFFERENTIAL OPERATORS}

As explained in the Introduction, one important application of integro-differential polynomials is the adjunction of new elements to an initially given integro-differential algebra $\mathcal{F}$; this issue will be broached in a future paper. If $\mathcal{F}$ is ordinary (see Definition 11 below), we can thus ensure that a given homogeneous differential equation $T u=0$ with monic $T \in \mathcal{F}[\partial]$ is dimensionally adequate, meaning $\operatorname{dim}_{K} \operatorname{Ker}(T)=\operatorname{deg} T$. This is the prerequisite for finding the Green's operator of the corresponding inhomogeneous equation $T u=f$; see [23] for a detailed description of the solution method. Its groundwork consists of adding and multiplying integro-differential operators, and this is what we shall consider here.

Before giving the construction of integro-differential operators, we will explicitly restrict ourselves to ordinary differential equations in the following sense. Note that in the following definition our terminology deviates from [15, p. 58], where it only refers to having a single derivation.

Definition 11. A differential algebra $\mathcal{F}$ over a field $K$ is called ordinary if $\operatorname{dim}_{K} \operatorname{Ker}(\partial)=1$. An integro-differential algebra $\left(\mathcal{F}, \partial, \int\right)$ is called ordinary if $(\mathcal{F}, \partial)$ is ordinary.

As a consequence, the solution space of a homogeneous differential equation $T u=0$ with monic $T \in \mathcal{F}[\partial]$ is now finite-dimensional, so we can indeed enforce dimensional adequacy by adjunction. (The notion of saturated integrodifferential algebra [23] postulates dimensional adequacy for every monic $T \in \mathcal{F}[\partial]$.)

Clearly we have $K=\mathcal{C}$ in an ordinary differential algebra $\mathcal{F}$, which is thus an algebra over its own field of constants. But then a section is automatically homogeneous over $\mathcal{C}$, so the pure Baxter axiom (4) and its differential version (5) coincide. Moreover, one knows from Linear Algebra that 
a projector $P$ onto a one-dimensional subspace $[w]$ of a $K$ vector space $V$ can be written as $P(v)=\varphi(v) w$ with a functional $\varphi$ that can be made unique by the normalization $\varphi(w)=1$. If $V$ is a $K$-algebra, a projector onto $K=[1]$ is canonically described by the functional $\varphi$ with $\varphi(1)=1$. This holds in particular in an ordinary differential algebra, where the projectors (3) corresponding to sections of the derivation can be regarded as normalized functionals.

In an ordinary integro-differential algebra $\mathcal{F}$, the normalized functional corresponding to the integral $\int$ is moreover multiplicative, as explained at the end of Section 2. We call this multiplicative functional

$$
\mathrm{E}=1-\int \partial
$$

its evaluation. The terminology stems from the standard example $\mathcal{F}=C^{\infty}[a, b]$, where $\mathrm{E}$ is a point evaluation (see below Definition 1). The multiplicative functionals on an algebra are known as its characters (note that all characters are normalized). We write $\mathcal{M}(\mathcal{F})$ for the vector space of all characters on an ordinary integro-differential algebra $\mathcal{F}$, including the evaluation $\mathrm{E}$ as a distinguished character.

Let $\mathcal{F}$ be a fixed ordinary integro-differential algebra over a field $K$ with evaluation $\mathrm{E}$. The variables $f, g$ are used for elements of $\mathcal{F}$, the variables $\varphi, \psi$ for elements of $\mathcal{M}(\mathcal{F})$. We introduce now an algebra of operators on $\mathcal{F}$ using rewrite systems [1] in the spirit of [3].

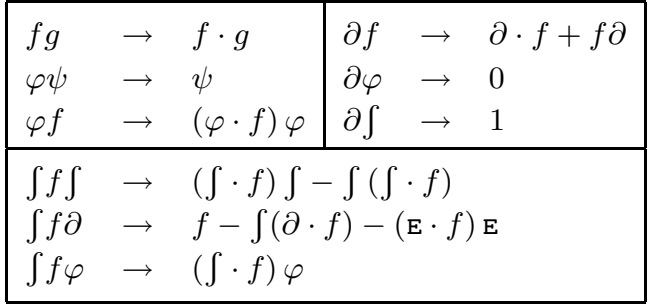

Table 1: Rewrite System for $\mathcal{F}\left[\partial, \int\right]$

Definition 12. The integro-differential operators $\mathcal{F}\left[\partial, \int\right]$ are defined as the $K$-algebra generated by the symbols $\partial$ and $\int$, the "functions" $f \in \mathcal{F}$ and the multiplicative "functionals" $\varphi \in \mathcal{M}(\mathcal{F})$, modulo the rewrite system of Table 1 .

In the rules of Table 1 , we use the notation $U \cdot f$ for the action of $U$ on an element $f \in \mathcal{F}$, where $U$ is an element of the free algebra in the above generators. It is an easy matter to check that the rewrite rules of Table 1 are fulfilled in $\mathcal{F}$, so we may lift - to an action of $\mathcal{F}\left[\partial, \int\right]$ on $\mathcal{F}$. In particular, $f \cdot g$ now denotes the product in $\mathcal{F}$.

We remark that Table 1 is to be understood as including implicit rules for $\iint, \int \partial$ and $\int \varphi$ by substituting $f=1$ in the rules for $\int f \int, \int f \partial$ and $\int f \varphi$, respectively. Moreover, one obtains the derived rule $\mathrm{E} \int=0$ from the definition of the evaluation E. Note also that $\mathcal{F}[\partial] \subseteq \mathcal{F}\left[\partial, \int\right]$, with the same induced action on $\mathcal{F}$.

TheOREM 13. The rewrite system for $\mathcal{F}\left[\partial, \int\right]$ in Table 1 is convergent.
In other words, the polynomials given by the difference between the left-hand and right-hand sides of Table 1 form a two-sided noncommutative Gröbner basis. The proof is given in [23]. For the theory of Gröbner bases, we refer to $[5,6]$, for its noncommutative extension to $[17,18]$.

\section{NORMAL FORMS FOR INTEGRO- DIFFERENTIAL OPERATORS}

Having a convergent rewrite system, every integro-differential operator has a unique normal form [1, p. 12]. To compute such normal forms we also need a canonical simplifier on the free algebra generated by $\partial$ and $\int$, the functions $f \in \mathcal{F}$ and the functionals $\varphi \in \mathcal{M}(\mathcal{F})$; one possibility is by basis expansion in $\mathcal{F}$. Here we summerize the description of the normal forms on $\mathcal{F}\left[\partial, \int\right]$ obtained in [23].

We first consider operators in the right ideal

$$
\mathcal{S}(\mathcal{F})=\mathcal{M}(\mathcal{F}) \mathcal{F}\left[\partial, \int\right],
$$

which we call Stieltjes boundary conditions over $\mathcal{F}$ or "boundary conditions" for short. Every such boundary condition has the normal form

$$
\sum_{\varphi \in \mathcal{M}(\mathcal{F})}\left(\sum_{i \in \mathbb{N}} a_{\varphi, i} \varphi \partial^{i}+\varphi \int f_{\varphi}\right)
$$

with $a_{\varphi, i} \in K$ and $f_{\varphi} \in \mathcal{F}$ almost all zero. We write $\mathcal{F}[\mathrm{E}]$ for the left $\mathcal{F}$-submodule generated by $\mathcal{S}(\mathcal{F})$ and call them Stieltjes boundary operators or "boundary operators" for short.

With the rule for $\partial f$ of Table 1 it is clear that the differential operators $\mathcal{F}[\partial] \subset \mathcal{F}\left[\partial, \int\right]$ have their usual normal forms. Analogously, we write $\mathcal{F}\left[\int\right] \subset \mathcal{F}\left[\partial, \int\right]$ for the subalgebra of integral operators, generated by the functions and $\int$ modulo the rule for $\int f \int$ of Table 1 ; one sees immediately that that their normal forms are linear combinations of $f \int g$ with $f, g \in \mathcal{F}$.

THEOREM 14. Up to ordering the summands, every normal form of $\mathcal{F}\left[\partial, \int\right]$ with respect to the rewrite system of Table 1 can be written uniquely as a sum $T+G+B$ with $T \in \mathcal{F}[\partial]$ and $G \in \mathcal{F}\left[\int\right]$ and $B \in \mathcal{F}[\mathrm{E}]$.

We can use integro-differential operators for specifying and solving boundary problems. Since space is limited, we can only state the main results here; for details and complete proofs, we must again refer to [23]. We formulate the boundary problem for a monic differential operator $T \in \mathcal{F}[\partial]$ with $\operatorname{deg} T=n$ and Stieltjes boundary conditions $\beta_{1}, \ldots, \beta_{n} \in$ $\mathcal{S}(\mathcal{F})$ as follows.

Given a forcing function $f \in \mathcal{F}$, find $u \in \mathcal{F}$ such that

$$
\begin{aligned}
& T u=f, \\
& \beta_{1} u=\ldots=\beta_{n} u=0 .
\end{aligned}
$$

We call the boundary problem regular if there is a unique $u \in$ $\mathcal{F}$ for every $f \in \mathcal{F}$; this implies in particular that $\beta_{1}, \ldots, \beta_{n}$ are linearly independent over $K$.

The first step in solving (17) is to consider the corresponding initial value problem based on a character $\eta \in \mathcal{M}(\mathcal{F})$, 
where one replaces the boundary conditions $\beta_{1}, \ldots, \beta_{n}$ by $\eta, \eta \partial, \ldots, \eta \partial^{n-1}$. Note that one may in particular choose $\eta=\mathrm{E}$, evaluating in the initialization point. The main idea of solving initial value problems is of course an adaption of the familiar variation-of-constants formula (see for example in $[9$, p. 74$]$ for systems and $[9$, p. 87$]$ for scalar differential equations).

Proposition 15. Let $T \in \mathcal{F}[\partial]$ be a monic differential operator with $\operatorname{deg} T=n$ such that $T u=0$ has a fundamental system of solutions $u_{1}, \ldots, u_{n} \in \mathcal{F}$. If $W$ is its Wronskian matrix and $d=\operatorname{det} W$ is invertible in $\mathcal{F}$, the initial value problem $T u=f$ based on $\eta \in \mathcal{M}(\mathcal{F})$ has the unique solution

$$
u=\sum_{i=1}^{n} u_{i}(1-\eta) \int d^{-1} d_{i} f
$$

for every forcing function $f \in \mathcal{F}$. Here $d_{i}=\operatorname{det} W_{i}$, where $W_{i}$ is the matrix obtained from $W$ by replacing the ith column by the nth unit vector.

Proof. We can use the usual technique of reformulating $T u=f$ as a system of linear first-order differential equations with companion matrix $A \in \mathcal{F}^{n \times n}$. The integral operator

$$
f=(1-\eta) \int
$$

is a section of $\partial$ with corresponding projector $1-f \partial=\eta$. Since $\eta$ is multiplicative, we know from Section 2 that $f$ is an integral. We extend the action of the operators $f, \partial, \eta$ componentwise to $\mathcal{F}^{n}$. Setting now

$$
\hat{u}=W f W^{-1} \hat{f}
$$

with $\hat{f}=(0, \ldots, 0, f)^{\top} \in \mathcal{F}^{n}$, one may readily check that $\hat{u} \in \mathcal{F}^{n}$ is a solution of the first-order system $\hat{u}^{\prime}=A \hat{u}+\hat{f}$ with initial condition $\eta \hat{u}=0$. Writing $u$ for the first component of $\hat{u}$, we have a solution of the initial value problem $T u=f$ based on $\eta \in \mathcal{M}(\mathcal{F})$. Using Cramer's rule to compute the $n$th column of $W^{-1}$, we see that

$$
W^{-1} \hat{f}=d^{-1} f\left(d_{1}, \ldots, d_{n}\right)^{\top},
$$

and (18) follows since the first row of $W$ is $\left(u_{1}, \ldots, u_{n}\right)$.

For proving uniqueness, assume $T u=0$ along with the initial conditions $\eta u=\ldots=\eta u^{(n-1)}=0$. Let

$$
u=c_{1} u_{1}+\ldots+c_{n} u_{n}
$$

with coefficients in $K$. Then the initial conditions yield $\eta(W c)=0$ with $c=\left(c_{1}, \ldots, c_{n}\right)^{\top} \in K^{n}$. But $\eta(W c)=$ $\eta(W) c$ because $\eta$ is linear, and $\operatorname{det} \eta(W)=\eta(\operatorname{det} W)$ because it is moreover multiplicative. Since $\operatorname{det} W \in \mathcal{F}$ is invertible, this implies that $\eta(W) \in K^{n \times n}$ is regular, so $c=\eta(W)^{-1} 0=0$ and $u=0$.

The above proposition hinges on two conditions: The first has already been discussed and can be satisfied by adjunction. The second condition needs an invertible Wronskian $d$. This could also be enforced by a suitable localization of $\mathcal{F}$, as for Picard-Vessiot rings [27, p. 12]. But in many applications, this condition will come out naturally: The Wronskian $d$ is always an exponential over $\mathcal{F}$ since it satisfies the differential equation $d^{\prime}=a d$, where $a$ is the trace of the companion matrix $A$.

Since every integro-differential algebra $\mathcal{F}$ comes with the evaluation $\eta=\mathrm{E}$ as a distinguished character, we can speak of the initial value problem associated with a monic $T \in$ $\mathcal{F}[\partial]$. Then the map $T^{\star}: \mathcal{F} \rightarrow \mathcal{F}$ described by the assignment $f \mapsto u$ in (18) simplifies to

$$
T^{\bullet}=\sum_{i=1}^{n} u_{i} \int d^{-1} d_{i} .
$$

We call $T^{\bullet} \in \mathcal{F}\left[\partial, \int\right]$ the fundamental right inverse of $T$. Note that (19) can be further simplified if $T$ has constant coefficients; see [22].

The next step in solving (17) is to compute the projector onto $\operatorname{Ker}(T)=\left[u_{1}, \ldots, u_{n}\right]$ along

$$
\left[\beta_{1}, \ldots, \beta_{n}\right]^{\perp}=\left\{u \in \mathcal{F} \mid \beta_{1} u=\ldots=\beta_{n} u=0\right\},
$$

which can be achieved as follows: Change from the basis $\beta_{1}, \ldots, \beta_{n}$ of $\left[\beta_{1}, \ldots, \beta_{n}\right]$ to a new basis $\tilde{\beta}_{1}, \ldots, \tilde{\beta}_{n}$ over $K$ biorthogonal to $u_{1}, \ldots, u_{n}$ in the sense that $\tilde{\beta}_{i}\left(u_{j}\right)=\delta_{i j}$. Then the projector can be determined as

$$
P=u_{1} \tilde{\beta}_{1}+\ldots+u_{n} \tilde{\beta}_{n} \in \mathcal{F}[\mathrm{E}] .
$$

See [23] for further details.

We can now put everything together for determining the Green's operator $G: f \mapsto u$ of (17). The point is that $T^{\diamond}$ solves the initial value problem, while $1-P$ "translates" the initial conditions $\mathrm{E}, \mathrm{E} \partial, \ldots, \mathrm{E} \partial^{n-1}$ to the required boundary conditions $\beta_{1}, \ldots, \beta_{n}$.

TheOREM 16. Let $T \in \mathcal{F}[\partial]$ be monic with $\operatorname{deg} T=n$ and $\beta_{1}, \ldots, \beta_{n} \in \mathcal{S}(\mathcal{F})$ such that the boundary problem (17) is regular. If the conditions of Proposition 15 are satisfied, the Green's operator of (17) is given by

$$
G=(1-P) T^{\star},
$$

where $P$ is the projector onto $\operatorname{Ker}(T)$ along $\left[\beta_{1}, \ldots, \beta_{n}\right]^{\perp}$.

Proof. Let $u_{1}, \ldots, u_{n}$ be a fundamental system for $T$. We have $T G=T T^{\star}-P T^{\star}=1-0$ since $P$ annihilates $u_{1}, \ldots, u_{n}$. Thus $u=G f$ satisfies the differential equation $T u=f$ of (17).

For ensuring the boundary conditions of (17), we prove $\beta_{i} G=0$ for $i=1, \ldots, n$. But we have even $\beta_{i}(1-P)=0$ because $1-P$ projects onto $\left[\beta_{1}, \ldots, \beta_{n}\right]^{\perp}$.

In analysis, the Green's operator $G$ is usually written as an integral operator with the bivariate Green's function as its kernel. As remarked in Section 2, this is the effect of the Baxter axiom. Hence the abstract version of a Green's function is the Green's operator $G \in \mathcal{F}\left[\partial, \int\right]$ written in its normal form. In the classical $C^{\infty}[a, b]$ setting, there is indeed a straight-forward correspondence between normal forms and Green's functions [22]. 


\section{CONCLUSION}

We have presented two algorithmic tools for studying integration from an algebraic operator perspective. The integrodifferential polynomials, introduced for the first time in this paper, enjoy a rich structure that deserves further analysis. Specifically, their quotient algebras are relevant in view of adjunctions (see at the beginning of Section 5). Unlike the integro-differential polynomials, the integro-differential operators are an algebraic model of linear operators, based on the (noncommutative) compositional structure. Their normal forms are much easier to describe since one can fall back on Gröbner basis methods. We can benefit from both in the study of differential equations - particularly when considered with boundary conditions.

\section{Acknowledgments}

We would like to thank our project leaders Bruno Buchberger and Heinz W. Engl for their continuous support, critical comments and helpful suggestions.

\section{REFERENCES}

[1] F. Baader and T. Nipkow. Term rewriting and all that. Cambridge University Press, Cambridge, 1998.

[2] G. Baxter. An analytic problem whose solution follows from a simple algebraic identity. Pacific J. Math., 10:731-742, 1960.

[3] G. M. Bergman. The diamond lemma for ring theory. Adv. in Math., 29(2):178-218, 1978.

[4] A. H. Bilge. A REDUCE program for the integration of differential polynomials. Comput. Phys. Comm., 71(3):263-268, 1992.

[5] B. Buchberger. An algorithm for finding the bases elements of the residue class ring modulo a zero dimensional polynomial ideal (German). PhD thesis, Univ. of Innsbruck, 1965. English translation published in J. Symbolic Comput., 41(3-4):475-511, 2006.

[6] B. Buchberger. Introduction to Gröbner bases. 1998. In [8], pp. 3-31.

[7] B. Buchberger and R. Loos. Algebraic simplification. In Computer algebra, pages 11-43. Springer, Vienna, 1983.

[8] B. Buchberger and F. Winkler, editors. Gröbner bases and applications, volume 251 of London Mathematical Society Lecture Note Series. Cambridge University Press, Cambridge, 1998. Papers from the Conference on 33 Years of Gröbner Bases held at the University of Linz, Linz, February 2-4, 1998.

[9] E. A. Coddington and N. Levinson. Theory of ordinary differential equations. McGraw-Hill Book Company, Inc., New York-Toronto-London, 1955.

[10] I. M. Gelfand and L. A. Dikiur. Fractional powers of operators, and Hamiltonian systems. Funkcional. Anal. i Priložen., 10(4):13-29, 1976. English translation: Functional Anal. Appl. 10 (1976), no. 4, 259-273 (1977).

[11] L. Guo. Baxter algebras and differential algebras. In Differential algebra and related topics (Newark, NJ, 2000), pages 281-305. World Sci. Publ., River Edge, NJ, 2002.

[12] L. Guo and W. Keigher. On differential Rota-Baxter algebras. J. Pure Appl. Algebra, 212(3):522-540, 2008.
[13] H. Hule. Polynome über universalen Algebren. Monatsh. Math., 73:329-340, 1969.

[14] I. Kaplansky. An introduction to differential algebra. Publ. Inst. Math. Univ. Nancago, No. 5. Hermann, Paris, 1957.

[15] E. Kolchin. Differential algebra and algebraic groups, volume 54 of Pure and Applied Mathematics. Academic Press, New York-London, 1973.

[16] H. Lausch and W. Nöbauer. Algebra of polynomials, volume 5 of North-Holland Mathematical Library. North-Holland Publishing Co., Amsterdam, 1973.

[17] F. Mora. Groebner bases for non-commutative polynomial rings. In AAECC-3: Proceedings of the $3 \mathrm{rd}$ International Conference on Algebraic Algorithms and Error-Correcting Codes, pages 353-362, London, UK, 1986. Springer-Verlag.

[18] T. Mora. An introduction to commutative and noncommutative Gröbner bases. Theoret. Comput. Sci., 134(1):131-173, 1994. Second International Colloquium on Words, Languages and Combinatorics (Kyoto, 1992).

[19] M. Z. Nashed and G. F. Votruba. A unified operator theory of generalized inverses. In M. Z. Nashed, editor, Generalized inverses and applications (Proc. Sem., Math. Res. Center, Univ. Wisconsin, Madison, Wis., 1973), pages 1-109. Academic Press, New York, 1976.

[20] R. Ree. Lie elements and an algebra associated with shuffles. Ann. of Math. (2), 68:210-220, 1958.

[21] G. Regensburger and M. Rosenkranz. An algebraic foundation for factoring linear boundary problems. Ann. Mat. Pura Appl. (4), 2008. DOI:10.1007/s10231-008-0068-3.

[22] M. Rosenkranz. A new symbolic method for solving linear two-point boundary value problems on the level of operators. J. Symbolic Comput., 39(2):171-199, 2005.

[23] M. Rosenkranz and G. Regensburger. Solving and factoring boundary problems for linear ordinary differential equations in differential algebras. $J$. Symbolic Comput., 2007. DOI:10.1016/j.jsc.2007.11.007.

[24] G.-C. Rota. Baxter algebras and combinatorial identities (I, II). Bull. Amer. Math. Soc., 75:325-334, 1969.

[25] G.-C. Rota. Ten mathematics problems I will never solve. Mitt. Dtsch. Math.-Ver., (2):45-52, 1998.

[26] I. Stakgold. Green's functions and boundary value problems. John Wiley \& Sons, New York, 1979.

[27] M. van der Put and M. F. Singer. Galois theory of linear differential equations, volume 328 of Grundlehren der Mathematischen Wissenschaften. Springer, Berlin, 2003. 